\title{
Nanomedicines for Infectious Diseases
}

\author{
Admire Dube ' (10)
}

Received: 4 March 2019 / Accepted: 4 March 2019 / Published online: 11 March 2019

(C) Springer Science+Business Media, LLC, part of Springer Nature 2019

Infectious diseases continue to pose a significant threat to global health. Over 200 infectious diseases are currently known to man. Fortunately, only a handful are responsible for significant morbidity and mortality. HIV/AIDS, tuberculosis (TB) and malaria top the list of deadly infectious diseases, and worldwide, these three diseases combined, are responsible for over 3 million deaths every year (1). Outbreaks of infectious diseases are also not uncommon and examples in recent history include the Ebola virus, Zika virus and Avian flu outbreaks which originated in West Africa, South America and Asia, respectively, before spreading to other parts of the world (2-4). This further emphasizes the global concern over infectious diseases.

Infectious diseases are distinguished in that a pathogen/ causative organism is responsible for the disease, and the pathogen is transmitted to humans, by other humans, animals or other transmitting agents such as insects (5). In several cases, the pathogen is primarily resident within the intracellular space, particularly of cells of the immune system, such as macrophages, which serve in the primary defence against the pathogen. The pathogen and the host cell are therefore the main target for nanomedicines designed for treatment of infectious diseases. Typically, nanomedicines are designed to enhance the cellular targeting, uptake and pharmacokinetics (PK) and pharmacodynamics (PD) of anti-infective agent(s) (drugs), achieve localized treatment, and to achieve immunotherapy (immune system engineering towards pathogen eradication).

By and large, the field of nanomedicine has been focused on treatment of cancer and other non-communicable diseases. However, in recent times we have seen increasing research efforts directed towards developing nanomedicines for

Admire Dube

adube@uwc.ac.za

Discipline of Pharmaceutics, School of Pharmacy, University of the Western Cape, Cape Town, South Africa treatment of infectious diseases. This is the primary reason for the production of this topical issue, which is intended to highlight work at the frontier of this 'emerging' field with a focus on TB, HIV/AIDS and malaria.

In this issue, bacteriomimetic nanoparticles of metalorganic frameworks (MOFs) were synthesized by Guo et al. (6). The interaction of MOF particles with macrophages was characterized since several infectious disease pathogens are resident in macrophages. This study provides a platform for future work in the development of MOF based therapeutic systems for TB and other infectious diseases. The dependence on shape and surface functionalization on the uptake of bacteriomimetic poly(lactic-co-glycolic acid) (PLGA) nanoparticles by HEp-2 epithelial cells is reported by Castoldi et al. (7). The authors also report the efficacy of the PLGA nanoparticles (loaded with gentamicin) against Shigella flexneri infected HEp-2 cells. Kutscher et al. report the development of an in vitro dynamic pharmacokinetic cell (macrophage) culture system with the aim of precise simulation of drug elimination profiles in human. Rifampicin loaded PLGA based nanoparticles were used in the model (8). Such a PK model is intended to support translational studies to develop nanomedicines for infectious diseases.

Given that drugs for infectious diseases are commonly administered as a cocktail of drugs, Wang et al. developed a liposomal formulation for co-delivery of colistin and ciprofloxacin (9). Colistin delivery is important from the perspective that this drug is currently considered the last line of defence against multidrug resistant Pseudomonas infections.

The number of cases of cutaneous TB is significant across the world. Burger et al. developed nano-emulsions, for the topical treatment of cutaneous TB, incorporating clofazimine and new drug combinations for $\mathrm{TB}$, i.e. artemisone and decoquinate (10). In tape stripping studies performed on human skin, the nano-emulsions were effective at delivering the drugs within the stratum corneum and epidermis. In this study, intracellular efficacy of the drug combinations was also 
demonstrated using Mycobacterium tuberculosis H37Rv J774 infected macrophages (10).

Mvango et al. (11) provide a review of the application of polymer therapeutics in treatment of infectious diseases. To date most of the polymer therapeutics have been applied to delivery of anti-cancer drugs. However, in this review, the authors discuss opportunities for polymer-drug conjugates for malaria treatment (and to some extent HIV/AIDS treatment). Grotz et al. present a review of the application of nanomedicine for the treatment of TB (12). Particular emphasis is placed on TB pharmacotherapy and how drug PK and PD shortcomings can be addressed using nanoparticles. Lastly, recent efforts to develop nanoparticle based immunotherapies for TB treatment and vaccination are highlighted in a review by Bekale et al. (13).

It is hoped readers will enjoy reading the articles and that they will be inspired to conduct work in this field. There is much scope for additional studies and translation of the technologies, as highlighted in the articles in this issue. Much appreciation and thanks is extended to the authors for their contributions.

\section{ACKNOWLEDGEMENTS AND DISCLOSURES}

The author acknowledges funding from the Fogarty International Center of the National Institutes of Health under Award Number K43TW010371. The content is solely the responsibility of the author and does not necessarily represent the official views of the National Institutes of Health.

Publisher's Note Springer Nature remains neutral with regard to jurisdictional claims in published maps and institutional affliations.

\section{REFERENCES}

1. World Health Organization. World health statistics 2018: monitoring health for the SDGs, sustainable development goals. Available from: https://apps.who.int/iris/bitstream/handle/10665/ 272596/9789241565585-eng.pdf?ua=1. Accessed 1 Mar 2019

2. Brasil P, Calvet GA, Siqueira AM, Wakimoto M, de Sequeira PC, Nobre A, et al. Zika virus outbreak in Rio de Janeiro, Brazil: clinical characterization, epidemiological and Virological aspects. PLoS Negl Trop Dis. 2016;10(4):e0004636.

3. Xie Y, Luo X, He Z, Zheng Y, Zuo Z, Zhao Q et al. VirusMap: a visualization database for the influenza a virus. J Genet Genomics. 2017;44(5):281-84

4. Spengler JR, Ervin ED, Towner JS, Rollin PE, Nichol ST. Perspectives on West Africa Ebola virus disease outbreak, 20132016. Emerg Infect Dis. 2016;22(6):956-63.

5. Nash AA, Dalziel RG, Fitzgerald JR. Chapter 1 - general principles. In: Mims' pathogenesis of infectious disease. 6th ed. Boston: Academic Press; 2015. p. 1-7.

6. Guo A, Durymanov M, Permyakova A, Sene S, Serre C, Reineke J. Metal organic framework (MOF) particles as potential bacteriamimicking delivery Systems for Infectious Diseases: characterization and cellular internalization in alveolar macrophages. Pharm Res. 2019;36(4):53.

7. Castoldi A, Empting M, De Rossi C, Mayr K, Dersch P, Hartmann $\mathrm{R}$, et al. Aspherical and spherical InvA497-functionalized Nanocarriers for intracellular delivery of anti-infective agents. Pharm Res. 2018;36(1):22.

8. Kutscher HL, Morse GD, Prasad PN, Reynolds JL. In vitro pharmacokinetic cell culture system that simulates physiologic drug and nanoparticle exposure to macrophages. Pharm Res. 2019;36(3):44.

9. Wang S, Yu S, Lin Y, Zou P, Chai G, Yu HH, et al. Co-delivery of ciprofloxacin and Colistin in liposomal formulations with enhanced in vitro antimicrobial activities against multidrug resistant Pseudomonas aeruginosa. Pharm Res. 2018;35(10):187.

10. Burger C, Aucamp M, du Preez J, Haynes RK, Ngwane A, du Plessis J, et al. Formulation of natural oil Nano-emulsions for the topical delivery of Clofazimine, Artemisone and Decoquinate. Pharm Res. 2018;35(10):186.

11. Mvango S, Matshe WMR, Balogun AO, Pilcher LA, Balogun MO. Nanomedicines for malaria chemotherapy: encapsulation vs. polymer therapeutics. Pharm Res. 2018;35(12):237.

12. Grotz E, Tateosian N, Amiano N, Cagel M, Bernabeu E, Chiappetta DA, et al. Nanotechnology in tuberculosis: state of the art and the challenges ahead. Pharm Res. 2018;35(11):213.

13. Bekale RB, Du Plessis S-M, Hsu N-J, Sharma JR, Sampson SL, Jacobs M, et al. Mycobacterium tuberculosis and interactions with the host immune system: opportunities for nanoparticle based Immunotherapeutics and vaccines. Pharm Res. 2018;36(1):8. 